\title{
Robust Optimization of Consumption with Random Endowment
}

\section{Wiebke Wittmüß*}

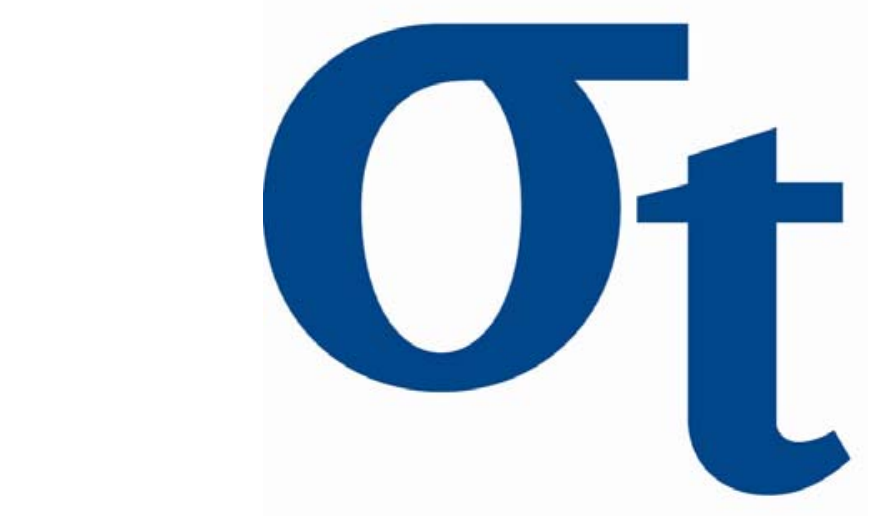

* Institute of Mathematics, Berlin University of Technology, Germany

This research was supported by the Deutsche Forschungsgemeinschaft through the SFB 649 "Economic Risk". 


\title{
Robust Optimization of Consumption with Random Endowment
}

July 21,2006

\author{
Wiebke Wittmüß* \\ Institute of Mathematics, MA 7-4 \\ Berlin University of Technology \\ Strasse des 17. Juni 136 \\ 12623 Berlin, Germany
}

\begin{abstract}
We consider the problem of optimal consumption for an investor who is risk and uncertainty avers. We model these preferences of the investor with the help of a convex risk-measure. Apart from consumption the agent has the possibility to invest initial capital and random endowment in a market where stock-prices are semimartingales. We formulate this as a maximin problem that will be solved by duality methods.
\end{abstract}

keywords: duality theory; risk measures; optimal consumption; model uncertainty

AMS classification 91B16, 62C20

JEL classification D11, D81

\section{Introduction}

Starting in 1969, 1971 with the works by Merton [19], [20] the problem of optimal investment has become one of the major research areas in mathematical finance. Merton and many of the following authors determined the optimal financial position with respect to expected utility but expected utility does not reflect preferences correctly in situations where model uncertainty is present. Therefore, Gilboa and Schmeidler [9] extended the model by incorporating Knightian

\footnotetext{
*Supported by Deutsche Forschungsgemeinschaft through the SFB 649 "Economic Risk".
} 
uncertainty which resulted in a robust approach. One possible interpretation of this approach is that the agent takes a class of possible models into consideration and decides for the worst-case outcome. This interpretation also explains why it is useful to extend the robust approach to an evaluation via risk measures: the agent will typically have some assessment of the different models (for instance she might use estimated data for the model specification and while she does not assume her estimates to be correct she believes values close to the estimates to be more likely than values far off). It is possible to include this assessment by the introduction of a penalty function, which allows to weight the impact of a model according to its plausibility. In Maccheroni et al. [18] a set of axioms is given yielding these preferences.

The non-robust problem of optimal terminal wealth was considered by several authors who discussed different aspects of the problem. For example Kramkov and Schachermayer [16], [17] characterized solutions of this problem in a general setting using duality methods. There exist many extensions of this problem, e.g. Cvitanić et al. [5], Karatzas and Zitkovic [15], or Hugonnier and Kramkov [14] consider the case where the agent optimizes the consumption process and also receives some kind of additional random endowment.

In [23], [25] and [10] the robust problem (without penalty term) was solved, also via the duality or martingale method. Furthermore, there are papers where the authors assume more specific market models and preferences and are therefore able to compute not just the optimal financial position but also the optimal investment strategy: See [7] for a stochastic control approach for a model with random endowment or [22] for computation of the optimal strategy in a robust setting. In [2] or [21] a optimal investment problem is solved using BSDE's. Also the robust problem with a penalty term was considered, among others by Hansen and Sargent [11], and Bordigoni et al.[3] for an entropic penalty term and by Hernández-Hernández and Schied [13] for a general penalty term.

In this paper we will investigate the problem of optimal consumption in a general semimartingale framework where the agent may invest in the stock market and receives additional random endowment. She evaluates positions by a robust utility functional described through a utility function $U$ and a penalty term $\gamma$. More specifically the agent tries to maximize

$$
\inf _{Q \ll P}\left(E_{Q}\left[\int_{0}^{T} U\left(t, c_{t}\right) \mu(d t)\right]+\gamma(Q)\right)
$$

over all possible consumption rate processes $c$. First the dual function for this problem is given and then we examine properties and relations between primal and dual problem, e.g. we will show 
that both problems have a solution and are conjugate to one another. Next we will verify that the above maximin problem and the corresponding minimax problem are equivalent.

In the long run we would like to compute explicitely the optimal consumption plan and investment strategy with the help of stochastic control methods (compare [13] or [12]). These techniques are - even in the nonrobust case - easier to apply to the dual problem than to the primal problem. In the robust case the primal problem becomes a maximin problem, i.e. we need to find a saddlepoint whereas the dual problem can still be solved through pure minimization. Hence, if we want to use stochastic control techniques it is sensible to examine the dual problem first. Furthermore, the duality results are also valid for preferences that are not dynamically consistent.

The problem is motivated by [24] where Schied considers the problem of optimal terminal wealth with respect to a convex risk measure in a setting without random endowment. We extend this problem by introducing a concept of consumption, general enough to include also the maximization of terminal wealth, and additional random endowment (compare [15]). In contrast to the problem of optimal terminal wealth, the problem of optimal consumption does not admit an equivalent static version. Hence, we cannot restrict ourselves to random variables to solve the primal problem, but need to work with stochastic processes. Furthermore, our dual problem is different from the dual problem in [24] or [15] due to random endowment on the one hand and robustification on the other. These differences imply for instance that there are cases where, given the dual solution $(\widehat{Q}, \widehat{R}), \widehat{R}$ is no longer a solution to the associated dual problem under the model $\widehat{Q}$. Aspects regarding the dual function will be discussed in more detail in the third section. Robustification changes the problem of optimal consumption also significantly with respect to the optimization procedure since we consider a maximin instead of just a maximization problem. This extension yields some new results and partly we give new proofs for known results.

In [4] Burgert and Rüschendorf also consider a robust version of [15]. The most obvious difference to our setting is that we deal with preferences given by convex instead of just coherent risk measures. Furthermore Burgert and Rüschendorf work under the serious restriction that the set $\mathcal{Q}$ should only consist of measures that are equivalent to $P$ and have a uniformly bounded density. The first assumption rules out risk measures such as AVaR, the second standard dynamic consistent coherent utility functionals in a Brownian setting such as used in [13].

In the next section we will introduce the market setting and describe the agent's preferences in more detail before we state our main theorem and give an example. In the third section we will derive our dual problem and prove the statements of the theorem related to it. Finally, in the 
fourth section we will finish the proof of the theorem.

\section{Notations and main results}

We consider an agent who wants to maximize her utility from consumption between time zero and some finite time horizon $T$. She is endowed with an initial capital and receives additional random endowment over time, which she may invest into $d$ assets. To formalize this problem we use the same market model as Karatzas and Žitković [15]. That means we model the price process of the assets as a $d$-dimensional RCLL semi-martingale on $\left(\Omega, \mathcal{F},\left(\mathcal{F}_{t}\right)_{0 \leq t \leq T}, P\right)$ where the filtration satisfies the usual conditions. The financial market is assumed to be arbitrage-free in the sense that the set $\mathcal{M}$ of supermartingale measures equivalent to $P$ is not empty. The portfolio process is denoted by $\theta=\left(\theta_{t}\right)_{0 \leq t \leq T}$, and we allow only those strategies for which $\int_{0}^{t} \theta_{u} d S_{u}$ is bounded from below by some constant. ${ }^{1}$ The initial capital is denoted by $x$ and the random endowment is described as a nondecreasing, adapted, RCLL process $\mathcal{E}=\left(\mathcal{E}_{t}\right)_{0 \leq t \leq T}$ with $\mathcal{E}_{T} \in L^{\infty}(P)$. The consumption process $C=\left(C_{t}\right)_{0 \leq t \leq T}$ is assumed to be a nonnegative, nondecreasing and adapted RCLL process. More specifically we will only deal with consumption processes that can be written as $C_{t}=\int_{0}^{t} c_{s} \mu(d s)$ where $\mu$ is a probability measure on $[0,1]$ which is diffuse on $[0,1)$. This means we assume that the agent consumes in a continuous way except for the final time $T$ where we also allow for lump consumption. In particular we can choose $\mu=\delta_{\{T\}}$ which accords to the problem of optimizing terminal wealth.

The terminal wealth is required to be non-negative:

$$
x+\mathcal{E}_{T}+\int_{0}^{T} \theta_{t} d S_{t}-C_{T} \geq 0 \quad P-\text { a.s. }
$$

We denote by $\mathcal{A}(x, \mu)$ the set of consumption process densities $\left(c_{t}\right)_{0 \leq t \leq T}$, for which an admissible strategy exists, such that condition (1) is fulfilled.

The utility from consumption is measured in terms of a robust utility functional of the form

$$
c \longmapsto \inf _{Q \ll P}\left(E_{Q}\left[\int_{0}^{T} U\left(t, c_{t}\right) \mu(d t)\right]+\gamma(Q)\right) .
$$

This approach is called robust since such utility functionals are useful to deal with model uncertainty $[9,18]$. The penalty function $\gamma$ enables us to influence how serious a model should be taken. The

\footnotetext{
${ }^{1}$ This condition will correspond to our admissibility condition for strategies, since the additional income of the agent is bounded.
} 
robust functional is closely linked to the convex risk measure

$$
\rho(Y):=\sup _{Q \ll P}\left(E_{Q}[-Y]-\gamma(Q)\right), \quad Y \in L^{\infty}(P) .
$$

More precisely the penalty function $\gamma$ is supposed to be bounded from below and equal to the minimal penalty function of the convex risk measure above, which means, that it satisfies the biduality relation

$$
\gamma(Q)=\sup _{Y \in L^{\infty}(P)}\left(E_{Q}[-Y]-\rho(Y)\right)
$$

Furthermore we need the following conditions on $\gamma$; compare [24, Assumption 2.1].

\section{Assumption 2.1}

We assume that the risk measure $\rho$ is continuous from below, i.e. for a sequence $\left(Y_{n}\right) \subset L^{\infty}$ increasing a.s. to some $Y \in L^{\infty}$, we have $\rho\left(Y_{n}\right) \searrow \rho(Y)$. Furthermore, $\rho$ needs to be sensitive in the sense that $\rho(Y)$ is strictly positive for all $Y \in L_{-}^{\infty} \backslash\{0\}$.

We work with a utility function $U:[0, T] \times \mathbb{R}^{+} \rightarrow \mathbb{R}$ with the properties assumed in Definition 3.1 of $[15]$.

\section{Assumption 2.2}

More precisely, for fixed $t \in[0, T]$ we request $U(t,):. \mathbb{R}^{+} \rightarrow \mathbb{R}$ to be a utility function, i.e. $U(t, \cdot)$ is strictly concave, increasing, continuously differentiable and satisfies the Inada conditions.

The marginal utility is assumed to be bounded by the strictly decreasing continuous functions $K_{1}$ and $K_{2}$, such that $K_{1}(x) \leq U_{x}(t, x) \leq K_{2}(x)$ and

$$
\limsup _{x \rightarrow \infty} K_{2}(x) / K_{1}(x)<\infty .
$$

The map $t \mapsto U(t, 1)$ is bounded and

$$
\lim _{x \rightarrow \infty} \inf _{t \in[0, T]} U(t, x)>0 .
$$

Additionally $U$ is supposed to be of reasonable asymptotic elasticity, i.e.

$$
\limsup _{x \rightarrow \infty}\left(\sup _{t} \frac{x U_{x}(t, x)}{U(t, x)}\right)<1
$$

See [15] for a discussion of these assumptions.

To avoid problems of evaluating $E_{Q}\left[\int_{0}^{T} U\left(t, c_{t}\right) \mu(d t)\right]+\gamma(Q)$ for the case where $U$ is unbounded from below it is sensible to restrict the set of measures that enter the optimization problem to $\mathcal{Q}=\{Q \ll P \mid \gamma(Q)<\infty\}$. Hence, our optimization problem is now

$$
\operatorname{maximize} \inf _{Q \in \mathcal{Q}}\left(E_{Q}\left[\int_{0}^{T} U\left(t, c_{t}\right) \mu(d t)\right]+\gamma(Q)\right) \text { over all } c \in \mathcal{A}(x, \mu) \text {. }
$$


Furthermore, to circumvent difficulties when integrating we follow [24] in setting

$$
E_{Q}[F]:=\sup _{n} E_{Q}[F \wedge n]=\lim _{n} E_{Q}[F \wedge n] \quad \text { for arbitrary } F \in L^{0} .
$$

In doing so we keep the functional $\left(c_{t}\right)_{0 \leq t \leq T} \longmapsto E_{Q}\left[\int_{0}^{T} U\left(t, c_{t}\right) \mu(d t)\right]$ concave.

In the following we will use $\mathcal{U}_{Q}(c)$ to abbreviate $E_{Q}\left[\int_{0}^{T} U\left(t, c_{t}\right) \mu(d t)\right]$. We define the value function of the maximization problem as follows

$$
u(x)=\sup _{c \in \mathcal{A}(x, \mu)} \inf _{Q \in \mathcal{Q}}\left(\mathcal{U}_{Q}(c)+\gamma(Q)\right) .
$$

Hence, the investor needs to solve a maximin problem in order to find $u$. Another approach to the optimization problem is to solve the problem in each possible model, i.e. compute $u_{Q}(x)=$ $\sup _{c \in \mathcal{A}(x, \mu)} \mathcal{U}_{Q}(c)$ for each $Q \in \mathcal{Q}$ and then robustify the result by taking the infimum. We will show that both methods lead to the same result, namely

$$
u(x)=\inf _{Q \in \mathcal{Q}}\left(u_{Q}(x)+\gamma(Q)\right)
$$

Sometimes it will be more convenient to work with densities than measures. We denote the density of $Q$ with respect to $P$ on $\mathcal{F}_{T}$ by $Z^{Q}=d Q / d P$ and the set $\left\{Z^{Q} \mid Q \in \mathcal{Q}\right\}$ by $\mathcal{Z}$. We will identify $Q$ and $Z^{Q}$, and thus write $\gamma\left(Z^{Q}\right) \gamma(Q)$ and $\gamma\left(Z^{Q}\right)$ or $u_{Q}$ and $u_{Z^{Q}}$ denote the same object. While we write $Z^{Q}$ for $d Q /\left.d P\right|_{\mathcal{F}_{T}}$ we will denote the corresponding density process by $\left(Z_{t}^{Q}\right)_{0 \leq t \leq T}$.

We will use the dual (or martingale) approach to characterize the solution to our optimization problem. A downside of the dual method is that it does not directly give the investment strategy $\theta$ which is necessary in order to realize the optimal consumption plan. In some cases this strategy can be determined using stochastic control techniques, compare e.g. [13] or [22]. These authors also argue that the dual problem is easier to treat than the primal problem which shows that our results are still useful. Furthermore, in our case we did not restrict the form of the penalty function, therefore our approach covers also the cases where time consistency is lacking and hence optimal control techniques cannot be applied (see [24]).

In general the dual problem is an associated minimization problem where the dual domain is related to the set of equivalent martingale measures. As was shown in [5] and [15] we need to use $\mathcal{D}$, the weak ${ }^{*}$-closure of the set of equivalent supermartingale measures $\mathcal{M}$, as dual domain. More precisely, we identify the set $\mathcal{M}$ with its embedding in the dual of $L^{\infty}(P),\left(L^{\infty}(P)\right)^{*}$. Then $\mathcal{D}$ is the $\sigma\left(\left(L^{\infty}(P)\right)^{*}, L^{\infty}(P)\right)$-closure of $\mathcal{M}$. The set $\mathcal{D}$ contains also finitely-additive measures to which we cannot directly associate a density process. Therefore, we use that each $R$ in $\mathcal{D}$ has a unique 
Hewitt-Yosida decomposition $R^{r}+R^{s}$ where the regular part $R^{r}$ is the maximal countable measure on $\mathcal{F}$ that is dominated by $R$. Hence, we can for each $R \in \mathcal{D}$ define a supermartingale $L^{R}$, where $L_{t}^{R}$ is the density of the regular part $\left(\left.R\right|_{\mathcal{F}_{t}}\right)^{r}$ of $\left.R\right|_{\mathcal{F}_{t}}$ with respect to $\left.P\right|_{\mathcal{F}_{t}}$. In the following we will work with the RCLL supermartingale $Y^{R}$ that coincides with $L^{R}$ for all $t \in \mathbb{Q} \cap[0, T]$. For a proof of the existence of $Y^{R}$ and further properties of $Y$ and $\mathcal{D}$ see [15]. We need as further notation $\left\langle R, \mathcal{E}_{T}\right\rangle$ which gives the canonical pairing. Observe that in particular $\left\langle R, I_{\Omega}\right\rangle=1$.

Using $\mathcal{D}$ we consider as dual problem

$$
v(y)=\inf _{Z \in \mathcal{Z}}\left(\inf _{R \in \mathcal{D}}\left(E\left[Z \int_{0}^{T} V\left(t, y \frac{Y_{t}^{R}}{Z_{t}}\right) \mu(d t)\right]+y\left\langle R, \mathcal{E}_{T}\right\rangle\right)+\gamma(Z)\right)
$$

where $V$ is the convex conjugate of $U$, i.e. $V(t, y)=\sup _{x \geq 0}(U(t, x)-x y)$. This problem results from the capital constraint (1). This link will be explained more explicitly in section 3 . To simplify notations, $E_{Q}\left[\int_{0}^{T} V\left(t, Y_{t}^{R}\right) \mu(d t)\right]$ will be denoted by $\mathcal{V}_{Q}\left(Y^{R}\right)$. We defined the function $u_{Q}$ as the solution to the optimization problem under the subjective probability $Q$. We can now define an associated dual value function $v_{Q}$ as

$$
v_{Q}(y)=\inf _{R \in \mathcal{D}}\left(\mathcal{V}_{Q}\left(y Y^{R} / Z^{Q}\right)+y\left\langle R, \mathcal{E}_{T}\right\rangle\right)
$$

\section{Remark 2.3}

In the introduction convex risk measures where interpreted as a worst-case approach for different scenarios $Q$. Using this interpretation it seems sensible to formulate the market restrictions under each measure $Q$ but we formulated the assumptions for the market and the trading strategy with respect to the measure $P$. (In particular the dual domain depends on the measure we use to give the capital constraint (1).) One reason why we use $P$ is that we use the convex risk measure as a model for the agent's preferences, not as a model of the "real" market. Furthermore, the worstcase measure may allow for arbitrage, compare [24, Example 3.2]. Hence, to exclude arbitrage opportunities it is necessary to formulate the admissibility condition under $P$.

Nevertheless, for all $Q$ out of $\mathcal{Q}_{e}:=\{Q \in \mathcal{Q} \mid Q \sim P\}$ we have the conditions necessary to apply standard duality results. (Lemma 3.3 will guarantee that $\mathcal{Q}_{e}$ is non-empty.)

\section{Assumption 2.4}

In the following we will assume that there exists $Q_{0} \in \mathcal{Q}_{e}$ that satisfies

$$
u_{Q_{0}}(x)<\infty \text { for some } x>0 \text {. }
$$

This is a similar assumption as is needed in [15] to guarantee the existence of solutions to both the primal and the dual problems under the subjective probability measure $Q_{0}$. Furthermore we 
can conclude that $u_{Q_{0}}$ and $v_{Q_{0}}$ are dual functions [15, Theorem 3.10]. We will show that we have similar results in our robust setting.

\section{Theorem 2.5}

Under the above assumption the following assertions are valid.

1. Both value functions $u$ and $v$ take only finite values and satisfy

$$
u^{\prime}(\infty-)=0 \quad \text { and } \quad v^{\prime}(0+)=-\infty
$$

$u$ is strictly concave and $v$ is continuously differentiable.

2. The value function $u$ satisfies

$$
u(x)=\sup _{c \in \mathcal{A}(x, \mu)} \inf _{Q \in \mathcal{Q}}\left(\mathcal{U}_{Q}(c)+\gamma(Q)\right)=\inf _{Q \in \mathcal{Q}}\left(\sup _{c \in \mathcal{A}(x, \mu)} \mathcal{U}_{Q}(c)+\gamma(Q)\right) .
$$

3. The two value functions $u$ and $v$ are conjugate to another:

$$
u(x)=\inf _{y>0}(v(y)+x y) \quad \text { and } \quad v(y)=\sup _{x>0}(u(x)-x y) .
$$

In particular, $v$ is convex.

4. The derivative of $v$ satisfies

$$
v^{\prime}(\infty-) \in\left[\inf _{R \in \mathcal{D}}\left\langle R, \mathcal{E}_{T}\right\rangle, \sup _{R \in \mathcal{D}}\left\langle R, \mathcal{E}_{T}\right\rangle\right] .
$$

If $\mathcal{E}_{T} \equiv 0$ the derivatives of $v$ and $u$ satisfy

$$
u^{\prime}(0+)=\infty \quad \text { and } \quad v^{\prime}(\infty-)=0 .
$$

5. There exists a solution $(\widehat{Q}, \widehat{R}) \in \mathcal{Q} \times \mathcal{D}$ to the dual problem, i.e.

$$
v(y)=\mathcal{V}_{\widehat{Q}, \mu}\left(y \frac{Y^{\widehat{R}}}{Z^{\widehat{Q}}}\right)+y\left\langle\widehat{R}, \mathcal{E}_{T}\right\rangle+\gamma(\widehat{Q}) .
$$

6. For any $x>0$ there exists an optimal consumption strategy $\hat{c} \in \mathcal{A}(x, \mu)$. If $\left(\widehat{Q}, Y^{\widehat{R}}\right)$ is a solution to the dual problem for $y>0$ such that $x=-v^{\prime}(y)$ then

$$
u(x)=\inf _{Q \in \mathcal{Q}}\left(\mathcal{U}_{Q}(\hat{c})+\gamma(Q)\right)=\mathcal{U}_{\widehat{Q}, \mu}(\hat{c})+\gamma(\widehat{Q})=u_{\widehat{Q}}(x)+\gamma(\widehat{Q})
$$

and

$$
\hat{c}=I\left(\cdot, \frac{\widehat{y} Y^{\widehat{R}}}{\widehat{Z}}\right) \quad \widehat{Q} \otimes \mu \text {-a.s. },
$$

where $\widehat{Z}=d \widehat{Q} / d P$ and $I(t, \cdot)=\left(\frac{\partial U}{\partial x}(t, \cdot)\right)^{-1}$. 
Obviously the problem is easier to treat if $\mathcal{Q}$ contains only measures that are equivalent to $P$. As in Schied [24] we get additional results in this case:

\section{Corollary 2.6}

If Assumption 2.4 and $\mathcal{Q}=\mathcal{Q}_{e}$ are satisfied and $\gamma$ is strictly convex on $\mathcal{Q}$ then the value function $u$ is continuously differentiable, the dual value function $v$ is strictly convex, and for each $y>0$ there exist $\widehat{Q} \in \mathcal{Q}$ and $\widehat{R} \in \mathcal{D}$ such that

$$
v(y)=\mathcal{V}_{\widehat{Q}, \mu}\left(y \frac{Y^{\widehat{R}}}{Z^{\widehat{Q}}}\right)+y\left\langle\widehat{R}, \mathcal{E}_{T}\right\rangle+\gamma(\widehat{Q}) .
$$

Moreover, $Y^{\widehat{R}}$ is unique. For any $x>0$, the optimal solution $\hat{c} \in \mathcal{A}(x, \mu)$ is $P$-a.s. unique.

(The condition of strict convexity for $\gamma$ is crucial for the differentiability of $u$.)

The following example considers the case of uncertain drift in a Black-Scholes model. Here one can see the advantage of a penalty function - if we used a purely robust approach the least absolute drift would always give the optimum but when a penalty is introduced the result is dependent on the drift under the measure $P$. For simplicity we consider a complete market. For computations in a more complex setting considerable additional effort is needed (compare e.g. [13]). Therefore we will treat these problems later. Nevertheless, we will then need the results from duality theory which we developed in this paper.

\section{Example 2.7}

We consider a Black-Scholes model, i.e. $S$ is given through $d S_{t}=S_{t}\left(b_{t} d t+\sigma d W_{t}\right)$ for constant $\sigma>0$ and As penalty function we take the relative entropy between $Q$ and $P$ and we look at the non time-dependent utility functional $U(t, x)=\log (x)$. If we do not restrict the market, we do not need to consider different processes $Y^{R}, R \in \mathcal{D}$ but only

$$
Y_{t}=\exp \left(-\int_{0}^{t} \frac{b_{s}}{\sigma} d W_{s}-\frac{1}{2} \int_{0}^{t} \frac{b_{s}^{2}}{\sigma^{2}} d s\right)
$$

(Compare [15, Proposition 4.1]). The convex conjugate of $U$ is given by $V(t, y)=-1-\log (y)$.

In [12, Lemma 3.1] it is proved that for each measure $Q \ll P$ we can write the density

$$
\frac{d Q}{d P}=\mathcal{E}\left(\int_{0}^{T} \eta_{s} d W_{s}\right) \quad Q-\text { a.s. }
$$

for a progressive $\eta$. We set $\tilde{b}_{s}=\sigma \eta_{s}+b_{s}$ which corresponds to the drift under $Q$. 
With this we have

$$
\begin{aligned}
v(y)= & \inf _{Z \in \mathcal{Z}}\left(E\left[Z \int_{0}^{T} V\left(t, y Y_{t} / Z_{t}\right) \mu(d t)\right]+\gamma(Z)\right) \\
= & \inf _{\tilde{b}}\left(E_{Q}\left[\int_{0}^{T} \log (1 / y)+\int_{0}^{t} \frac{\tilde{b}_{s}}{\sigma} d W_{s}^{Q}+\frac{1}{2} \int_{0}^{t} \frac{\tilde{b}_{s}^{2}}{\sigma^{2}} d s \mu(d t)\right]-1\right. \\
& \left.+E_{Q}\left[-\int_{0}^{T} \frac{b_{t}-\tilde{b}_{t}}{\sigma} d W_{t}-\frac{1}{2} \int_{0}^{T} \frac{\left(b_{t}-\tilde{b}_{t}\right)^{2}}{\sigma^{2}} d t\right]\right) .
\end{aligned}
$$

If we consider the case where $\mu=\delta_{\{T\}}$ we get

$$
v(y)=-E_{Q}\left[\int_{0}^{T} \frac{b_{t}}{\sigma} d W_{t}^{Q}\right]+2 E_{Q}\left[\int_{0}^{T} \frac{\tilde{b}_{t}}{\sigma} d W_{t}^{Q}\right]+E_{Q}\left[\int_{0}^{T} \frac{\left(b_{t}-\tilde{b}_{t}\right)^{2}+b_{t} \tilde{b}_{t}-\frac{1}{2} b_{t}^{2}}{\sigma^{2}} d t\right] .
$$

Hence, here the infimum is achieved for $\tilde{b}_{t}^{*}=b_{t} / 2$. Furthermore, one can calculate that

$$
u(x)=\log x+\frac{T b^{2}}{4 \sigma^{2}} .
$$

If we choose $\mu(d t)=d t$, take $b$ to be constant, and restrict the set $\mathcal{Z}$ to processes for which $\eta$ is also constant we get

$$
v(y)=\inf _{\tilde{b}}\left(E_{Q}\left[\int_{0}^{T} \log (1 / y)+\frac{1}{2} \int_{0}^{t} \frac{\tilde{b}_{s}^{2}}{\sigma^{2}} d s \mu(d t)\right]-1+E_{Q}\left[\frac{1}{2} \int_{0}^{T} \frac{\left(b_{t}-\tilde{b}_{t}\right)^{2}}{\sigma^{2}} d t\right]\right) .
$$

Which yields that the optimal $\tilde{b}$ is given by $\tilde{b}^{*}=2 b /(T+2)$. Obviously the drift that gives the infimum depends on the original drift $b$.

\section{Dual Problem}

We now develop the dual problem. The general approach is to use the capital constraint to bound the result of the primal problem from above which gives a related minimization problem. Karatzas and Žitković prove (Proposition 2.13 in [15]) that the capital constraint (1) can equivalently be formulated as follows, using the set $\mathcal{D}$ introduced above. The set $\mathcal{A}(x, \mu)$ of admissible consumption rates consists of all processes $c$ such that $\left(C_{t}:=\int_{0}^{t} c_{u} \mu(d u)\right)_{0 \leq t \leq T}$ is a nonnegative, nondecreasing, right-continuous and adapted process satisfying

$$
E\left[\int_{0}^{T} Y_{t}^{R} c_{t} \mu(d t)\right] \leq x+\left\langle R, \mathcal{E}_{T}\right\rangle \quad \text { for all } R \in \mathcal{D} .
$$


With the help of this characterization we can justify our dual problem by the next calculation. Due to (13) we have for all $y>0$ and all $R \in \mathcal{D}$

$$
\begin{aligned}
\mathcal{U}_{Q}(c) & \leq E_{Q}\left[\int_{0}^{T} U\left(t, c_{t}\right) \mu(d t)\right]+y\left(x+\left\langle R, \mathcal{E}_{T}\right\rangle-E\left[\int_{0}^{T} c_{t} Y_{t}^{R} \mu(d t)\right]\right) \\
& \leq E\left[Z^{Q} \int_{0}^{T} U\left(t, c_{t}\right)-y c_{t} \frac{Y_{t}^{R}}{Z_{t}^{Q}} \mu(d t)\right]+y\left(x+\left\langle R, \mathcal{E}_{T}\right\rangle\right) \\
& \leq E\left[Z^{Q} \int_{0}^{T} V\left(t, y \frac{Y_{t}^{R}}{Z_{t}^{Q}}\right) \mu(d t)\right]+y\left(x+\left\langle R, \mathcal{E}_{T}\right\rangle\right) .
\end{aligned}
$$

Hence, we arrive at the dual problem

$$
v(y)=\inf _{Z \in \mathcal{Z}}\left(\inf _{R \in \mathcal{D}}\left(E\left[Z \int_{0}^{T} V\left(t, y \frac{Y_{t}^{R}}{Z_{t}}\right) \mu(d t)\right]+y\left\langle R, \mathcal{E}_{T}\right\rangle\right)+\gamma(Z)\right) .
$$

\section{Remark 3.1}

When considering the inner problem we could also define

$$
\tilde{v}_{Q}(y)=\inf _{R \in \mathcal{D}_{Q}} E_{Q}\left[\int_{0}^{T} V\left(t, y Y_{t}^{R, Q}\right) \mu(d t)\right]+y\left\langle R, \mathcal{E}_{T}\right\rangle
$$

where $\mathcal{D}_{Q}$ is the weak*-closure of the set of supermartingale measures equivalent to $Q$ in $\left(L^{\infty}(P)\right)^{*}$ and $Y^{R, Q}$ is again defined as a version of the density process of the regular part of $R$ but here with respect to $Q$. Obviously $\mathcal{D}_{Q}$ can be empty if the market given by the measure $Q$ admits arbitrage. In this case we set the infimum to infinity. With the help of the functions $\left(\tilde{v}_{Q}\right)_{Q \in \mathcal{Q}}$ we can define an alternative dual function $\tilde{v}$ :

$$
\tilde{v}(y)=\inf _{Q \in \mathcal{Q}}\left(\tilde{v}_{Q}(y)+\gamma(Q)\right) .
$$

Schied defines his dual problem in [24] in this way. In contrast to our setting he is able to show that there is a one-to-one correspondence between the two kinds of dual sets [24, Lemma 4.2]. In our case no similar result holds, i.e. there is no way to decide whether $R$ belongs to $\mathcal{D}$ based on the knowledge of $c D_{Q}$. This is due to the random endowment. Nevertheless, we can still show that $v(y)=\tilde{v}(y)$ for all $y>0$. If we define the dual problem via the function $\tilde{v}$ there are cases where the infima are not attained in contrast to assertion 5 of Theorem 2.5. This will be illustrated by the example below.

\section{Example 3.2}

In this example we will have a unique solution $(\widehat{R}, \widehat{Q})$ to the dual problem for which $\widehat{R} \notin \mathcal{D}_{\widehat{Q}}$. We consider all convex combinations of two measures $Q^{*}$ and $P$, i.e. $\mathcal{Q}=\left\{Q^{\alpha}=\alpha P+(1-\alpha) Q^{*} \mid 0 \leq\right.$ $\alpha \leq 1\}$. The penalty function is given by $\gamma\left(Q^{\alpha}\right)=\alpha$, and $\gamma(Q)=\infty$ for all $Q \notin \mathcal{Q}$. Under 
$P, S_{0}=10$ and $S_{T}$ takes the values 5 and 15 each with probability 0.5 . Under $Q^{*}, S_{0}=10$ and $S_{T}=5 Q^{*}$-a.s. In order to achieve a continuous time model we set $S_{t}=S_{0}$ for all $t<T$. Obviously $Q^{*} \ll P$. Assume that $\mathcal{E}_{T}=m I_{\left\{S_{T}=5\right\}}, \mu=\delta_{T}$ and $U(t, x)=-e^{-x}$. Hence, $V(t, y)$ equals $y(\ln y-1)$ for $y \leq 1$ and -1 otherwise. Observe that $\mathcal{D}$ contains all measures $R$ that satisfy $\beta:=R\left[S_{T}=5\right] \geq 0.5$ and $\mathcal{D}_{Q^{*}}$ contains only $Q^{*}$. Then

$$
\begin{aligned}
v(y) & =\inf _{\alpha \in[0,1]} \inf _{R \in \mathcal{D}}\left(E_{Q^{\alpha}}\left[\int_{0}^{T} V\left(t, y Y_{t}^{R} / Z_{t}^{\alpha}\right) \mu(d t)\right]+\gamma(Q)+\left\langle R, \mathcal{E}_{T}\right\rangle\right) \\
& =\inf _{\alpha \in[0,1]} \inf _{\beta \in[1 / 2,1]}\left(\left(1-\frac{\alpha}{2}\right) V\left(T, y \frac{\beta}{1-\alpha / 2}\right)+\frac{\alpha}{2} V\left(T, y \frac{1-\beta}{\alpha / 2}\right)+\alpha n+\beta m\right) .
\end{aligned}
$$

We want to compute $v(1)$, hence we get different situations for $\beta<1-\alpha / 2$ and $\beta \geq 1-\alpha / 2$. First we compute the infima for the case $\beta<1-\alpha / 2$. Here we need to consider

$$
g(\alpha, \beta)=\left(1-\frac{\alpha}{2}\right) \frac{\beta}{1-\alpha / 2}\left(\ln \frac{\beta}{1-\alpha / 2}-1\right)-\frac{\alpha}{2}+\alpha n+\beta m .
$$

The partial derivatives of $g$ are

$$
g_{\alpha}(\alpha, \beta)=n-\frac{\alpha}{2}+\frac{\beta}{2(1-\alpha / 2)}
$$

and

$$
g_{\beta}(\alpha, \beta)=m+\ln \left(\frac{\beta}{1-\alpha / 2}\right)
$$

If we choose $m=1$ and $n=2$ both derivatives are positive and therefore the infimum for this case is reached for $\alpha=0$ and $\beta=1 / 2$, and takes the value $1 / 2(\ln 1 / 2-1)+1 / 2<0$. In the case $\beta \geq 1-\alpha / 2$ we need to compute the infimum of the function

$$
h(\alpha, \beta)=-(1-\alpha / 2)+\frac{\alpha}{2} \frac{1-\beta}{\alpha / 2}\left(\ln \frac{1-\beta}{\alpha / 2}-1\right)+\alpha n+\beta m .
$$

We have that

$$
\begin{aligned}
h(\alpha, \beta) & =-1+\alpha / 2+\frac{\alpha}{2} \frac{1-\beta}{\alpha / 2} \ln \frac{1-\beta}{\alpha / 2}-(1-\beta)+2 \alpha+\beta \\
& \geq-2+2 \beta+\left(2+1 / 2-1 / 2 e^{-1}\right) \alpha \\
& \geq-2+2(1-\alpha / 2)+\left(2+1 / 2-1 / 2 e^{-1}\right) \alpha>0 .
\end{aligned}
$$

For the first inequality we used the fact that $x \ln x \geq-e^{-1}$.

Hence, the solution to the dual problem is given by the first case, and equals $\left(Q^{0}, P\right)=\left(Q^{*}, P\right)$. Obviously $P \notin \mathcal{D}_{Q^{*}}$. 
In the rest of this section we will prove that in contrast to the example the solution to our dual problem exists, and that $v$ equals $\tilde{v}$. First we observe that for $Q_{0} \in \mathcal{Q}_{e}$ of Assumption $2.4[15$, Theorem 3.10] guarantees that $v_{Q_{0}}(y)<\infty$ for all $y>0$ and consequently also $v(y)<\infty$ for all $y>0$.We will repeatedly make use of a version of Komlós principle of convergence, compare e.g. [6, Lemma A1.1]. To control the behavior of the penalty function we will need the following lemma, which is taken from Schied [24, Lemma 4.1].

\section{Lemma 3.3}

For $d \geq 0$ denote the subsets of $\mathcal{Z}$ corresponding to

$$
\mathcal{Q}(d):=\{Q \in \mathcal{Q} \mid \gamma(Q) \leq d\} \quad \text { and } \quad \mathcal{Q}_{e}(d):=\{Q \in \mathcal{Q}(d) \mid Q \sim P\}
$$

by $\mathcal{Z}(d)$ and $\mathcal{Z}_{e}(d)$. Then for every $d>0$, the level set $\mathcal{Z}(d)$ is weakly compact, and $\mathcal{Z}_{e}(d)$ is nonempty. Moreover, $Z \mapsto \gamma(Z)$ is lower semicontinuous with respect to $P$-a.s. convergence on $\mathcal{Z}(d)$.

We will need the following technical result.

\section{Lemma 3.4}

For each constant $d>0$ the set,

$$
\left\{Z V^{-}\left(\cdot, \frac{y Y^{R}}{Z .}\right) \mid Z \in \mathcal{Z}(d), R \in \mathcal{D}\right\}
$$

is uniformly integrable with respect to $P \otimes \mu$.

Proof. Proposition 3.5 in [15] guarantees the existence of a utility function $\underline{U}$ such that $\underline{U}(x) \leq$ $U(t, x)$ for all $x>0$ and all $t \in[0, T]$. Furthermore, the convex conjugate $\underline{V}$ to $\underline{U}$ satisfies $\underline{V}(\cdot) \leq$ $V(t, \cdot)$ for all $0 \leq t \leq T$. Since $\mathcal{Z}(d)$ is uniformly integrable (according to Lemma 3.3 and the Dunford-Pettis theorem) the claim follows immediately if $\underline{V}$ is bounded from below. Assume $\underline{V}$ is not bounded from below. Let $\varphi$ denote the inverse function of $-\underline{V}$ and $y_{0}=\varphi(0)$. Then it follows that

$$
\begin{aligned}
E\left[\int_{0}^{T} Z_{t} \varphi\left(V^{-}\left(y \frac{Y_{t}^{R}}{Z_{t}}\right)\right) \mu(d t)\right] & \leq E\left[\int_{0}^{T} Z_{t} \varphi\left(-\underline{V}\left(y \frac{Y_{t}^{R}}{Z_{t}}\right)\right) \mu(d t)\right]+y_{0} \\
& \leq E\left[\int_{0}^{T} y Y_{t}^{R} \mu(d t)\right]+y_{0} \\
& \leq y+y_{0}=M .
\end{aligned}
$$


In [16] it was proved that $\varphi(h) / h \rightarrow \infty$ as $h \rightarrow \infty$. Hence for each $a$ there exists $d(a)$ such that $\varphi(h) \geq a h$ for all $h \geq d(a)$. Let $\varepsilon>0$ and take $d=d(2 M / \varepsilon), \eta=\varepsilon / 2 d$. If $A \in \mathcal{F} \otimes \mathcal{B}([0, T])$ with $E\left[\int_{0}^{T} Z_{t} I_{A} \mu(d t)\right]<\eta$ then we obtain the following inequality.

$$
\begin{aligned}
& E\left[\int_{0}^{T} Z_{t} V^{-}\left(t, \frac{y Y_{t}^{R}}{Z_{t}}\right) I_{A} \mu(d t)\right] \\
& \quad \leq E\left[\int_{0}^{T} Z_{t} \underline{V}^{-}\left(\frac{y Y_{t}^{R}}{Z_{t}}\right)\left(I_{A \cap\left\{\underline{V}^{-}\left(y Y_{t}^{R} / Z_{t}\right) \geq d\right\}}+I_{A \cap\left\{\underline{V}^{-}\left(y Y_{t}^{R} / Z_{t}\right)<d\right\}}\right) \mu(d t)\right] \\
& \quad \leq E\left[\int_{0}^{T} Z_{t} \varphi\left(\underline{V}^{-}\left(\frac{y Y_{t}^{R}}{Z_{t}}\right)\right) I_{A \cap\left\{\underline{V}^{-}\left(y Y_{t}^{R} / Z_{t}\right) \geq d\right\}} \mu(d t)\right] \frac{\varepsilon}{2 M}+d E\left[\int_{0}^{T} Z_{t} I_{A} \mu(d t)\right] \\
& \quad<\varepsilon
\end{aligned}
$$

Because of the uniform integrability of $\mathcal{Z}(d)$ there exists $\delta>0$ such that $E\left[\int_{0}^{T} Z_{t} I_{A} \mu(d t)\right]<\eta$ as soon as $(P \otimes \mu)[A]<\delta$. This finishes the proof.

The next lemma shows assertion 5 of the main theorem. In this and some later proofs we will need that the map $(x, y) \mapsto x V(t, y / x)$ is convex for all $0 \leq t \leq T$, and that

$$
\alpha x_{0} V\left(t, y_{0} / x_{0}\right)+(1-\alpha) x_{1} V\left(t, y_{1} / x_{1}\right)>\left(\alpha x_{0}+(1-\alpha) x_{1}\right) V\left(t, \frac{\alpha y_{0}+(1-\alpha) y_{1}}{\alpha x_{0}+(1-\alpha) x_{1}}\right)
$$

for $y_{1} / x_{1} \neq y_{0} / x_{0}$ and $\alpha \in(0,1)$; see e.g. [24, equation (25)].

\section{Lemma 3.5}

There exist $\widehat{Z} \in \mathcal{Z}$ and $\widehat{R} \in \mathcal{D}$ such that

$$
v(y)=E\left[\widehat{Z} \int_{0}^{T} V\left(t, y \frac{Y_{t}^{\widehat{R}}}{\widehat{Z}_{t}}\right) \mu(d t)\right]+y\left\langle\widehat{R}, \mathcal{E}_{T}\right\rangle+\gamma(\widehat{Z}) .
$$

Proof. Observe that Assumption 2.4 guarantees that $v(y)<\infty$ (compare Karatzas/Zitkovic [15, Theorem 3.10]). Let $\left(Z^{n}, R^{n}\right)_{n \in \mathbb{N}}$ be a sequence in $\mathcal{Z} \times \mathcal{D}$ such that

$$
E\left[Z^{n} \int_{0}^{T} V\left(t, y \frac{Y_{t}^{R^{n}}}{Z_{t}^{n}}\right) \mu(d t)\right]+y\left\langle R^{n}, \mathcal{E}_{T}\right\rangle+\gamma\left(Z^{n}\right) \rightarrow v(y) .
$$

Let $d Q^{n}=Z^{n} d P$, then Jensen's inequality and Fubini's theorem imply that

$$
\begin{aligned}
E\left[Z^{n} \int_{0}^{T} V\left(t, y \frac{Y_{t}^{R^{n}}}{Z_{t}^{n}}\right) \mu(d t)\right] & \geq E\left[Z^{n} \int_{0}^{T} \underline{V}\left(y \frac{Y_{t}^{R^{n}}}{Z_{t}^{n}}\right) \mu(d t)\right] \\
& \geq \int_{0}^{T} \underline{V}\left(E_{Q^{n}}\left[\frac{y Y_{t}^{R^{n}}}{Z_{t}^{n}}\right]\right) \mu(d t) \\
& =\int_{0}^{T} \underline{V}\left(E\left[y Y_{t}^{R^{n}} ; Z_{t}^{n}>0\right]\right) \mu(d t) \\
& \geq \underline{V}(y) .
\end{aligned}
$$


Since $\mathcal{E}_{T} \geq 0$ it follows that $d:=1+\lim \sup _{n \rightarrow \infty} \gamma\left(Z^{n}\right)<\infty$ and we may assume that $Z^{n} \in \mathcal{Z}(d)$ for all $n$.

Now we want to construct a sequence converging to the optimal $(\widehat{Z}, \widehat{R})$. For this we apply repeatedly a Komlós-type argument. Observe that in our case convex combinations of a converging sequence are still convergent. First we obtain a sequence $\left(\tilde{Z}^{n}, \tilde{R}^{n}\right)_{n}$ of convex combinations of $\left(Z^{n}, R^{n}\right)_{n}$, i.e. $\left(\tilde{Z}^{n}, \tilde{R}^{n}\right) \in \operatorname{conv}\left\{\left(Z^{n}, R^{n}\right),\left(Z^{n+1}, R^{n+1}\right), \ldots\right\}$, where $\tilde{Z}^{n}$ converges $P$ - a.s. to some $\widehat{Z} \in \mathcal{Z}(d)$ (compare Lemma 3.3). To get also convergence for the processes $\left(Z_{t}\right)_{0 \leq t \leq T}$ and $\left(Y_{t}^{R^{n}}\right)_{0 \leq t \leq T}$ we will argue analogously to the proof of Proposition A.2 in [15]. Due to [8, Lemma $5.2]$ we can choose a sequence of convex combinations $\left(\left(\widehat{Z}_{t}^{n}\right)_{0 \leq t \leq T},\left(Y_{t}^{\widehat{R}^{n}}\right)_{0 \leq t \leq T}\right)$ of elements in $\left.\left(\left(\tilde{Z}_{t}^{m}\right)_{0 \leq t \leq T}\right),\left(Y_{t}^{\tilde{R}^{m}}\right)_{0 \leq t \leq T}\right)_{m \geq n}$ that Fatou-converges to some RCLL supermartingale $\left(\widehat{Z}_{t}\right)_{0 \leq t \leq T}$. Due to Lemma 3.3 and the fact that $\mu$ is diffuse on $[0, T)$ we can find a subsequence that converges also $P \otimes \mu$-a.s. to $\left(\widehat{Z}_{t}\right)_{0 \leq t \leq T}$. We know that $\tilde{Z}=\widehat{Z}_{T} \in \mathcal{Z}(d)$ hence $\left(\widehat{Z}_{t}\right)_{0 \leq t \leq T}$ is a density process. To get similarly $Y^{\widehat{R}}$ we first extract a subsequence of $\left(\widehat{R}^{n}, \widehat{Z}^{n}\right)_{n}$ also denoted by $\left(\tilde{R}^{n}, \tilde{Z}^{n}\right)_{n}$ such that $\left\langle\widehat{R}^{n}, \mathcal{E}_{T}\right\rangle$ converges in $\mathbb{R}$. Then we consider the corresponding series $\left(Y^{\widehat{R}^{n}}\right)_{n \in \mathbb{N}}$. We have a sequence $\left(\tilde{Y}^{\widehat{R}^{n}}\right)_{n \in \mathbb{N}}$ of convex combinations of $\left(Y^{\widehat{R}^{n}}\right)$ converging $P \otimes \mu$ - a.s. to some $Y^{\widehat{R}}$ where $\widehat{R}$ is a weak* cluster point of $\widehat{R}^{n} \in \mathcal{D}$.

For $Z \in \mathcal{Z}(d), R \in \mathcal{D}$ the function $\left(Z, Y^{R}\right) \mapsto E\left[Z \int_{0}^{T} V\left(t, y Y_{t}^{R} / Z_{t}\right) \mu(d t)\right]$ is lower semicontinuous with respect to $P$-a.s. convergence. This can be proved the same way as Lemma 3.7 in [25] using Lemma 3.4. Combining this lower semicontinuity with Lemma 3.3 and the fact that $(x, y) \mapsto x V(t, y / x)$ is a convex function, results in

$$
\begin{aligned}
E\left[\widehat{Z} \int_{0}^{T}\right. & \left.V\left(t, y \frac{Y_{t}^{\widehat{R}}}{\widehat{Z}_{t}}\right) \mu(d t)\right]+y\left\langle\widehat{R}, \mathcal{E}_{T}\right\rangle+\gamma(\widehat{Z}) \\
& \leq \liminf _{n \rightarrow \infty}\left(E\left[Z^{n} \int_{0}^{T} V\left(t, y \frac{Y_{t}^{R^{n}}}{Z_{t}^{n}}\right) \mu(d t)\right]+y\left\langle R^{n}, \mathcal{E}_{T}\right\rangle+\gamma\left(Z^{n}\right)\right) \\
& =v(y) .
\end{aligned}
$$

This proves the optimality of $(\widehat{Z}, \widehat{R})$.

We will now show that we may replace the set $\mathcal{Q}$ in the dual problem by $\mathcal{Q}_{e}$ as well as $\mathcal{Q}_{e}^{f}$, and that the dual functions are equal. (Where $\mathcal{Q}_{e}^{f}$ denotes the set of measures $Q \in \mathcal{Q}_{e}$ where $u_{Q}(x)$ is finite for some $x>0$.) 


\section{Lemma 3.6}

The dual value function of the robust problem satisfies

$$
\tilde{v}(y)=v(y)=\inf _{Q \in \mathcal{Q}_{e}^{f}}\left(v_{Q}(y)+\gamma(Q)\right)=\inf _{Q \in \mathcal{Q}_{e}}\left(v_{Q}(y)+\gamma(Q)\right) .
$$

Proof. Let $\left(Z^{1}, R^{1}\right) \in \mathcal{Z} \times \mathcal{D}$ be such that

$$
v(y)=E\left[Z^{1} \int_{0}^{T} V\left(t, y Y_{t}^{R^{1}} / Z_{t}^{1}\right) \mu(d t)\right]+\gamma\left(Z^{1}\right)+\left\langle R^{1}, \mathcal{E}_{T}\right\rangle<\infty .
$$

Due to Assumption 2.4 and the assumptions on $U$ we can choose $Z^{0} \in \mathcal{Z}_{e}$ and $R^{0} \in \mathcal{D}$ such that also $E\left[Z^{0} \int_{0}^{T} V\left(t, y Y_{t}^{R^{0}} / Z_{t}^{0}\right) \mu(d t)\right]+\gamma\left(Z^{0}\right)<\infty$. Let $Z^{\alpha}:=\alpha Z^{1}+(1-\alpha) Z^{0} \in \mathcal{Z}_{e}$ and $R^{\alpha}=\alpha R^{1}+(1-\alpha) R^{0}$ for $0 \leq \alpha<1$. Since

$$
\alpha \mapsto E\left[Z^{\alpha} \int_{0}^{T} V\left(t, y Y_{t}^{R^{\alpha}} / Z_{t}^{\alpha}\right) \mu(d t)\right]
$$

is convex and takes only finite values it is upper semicontinuous. Moreover, with the same argument we can conclude that $\alpha \mapsto \gamma\left(Z^{\alpha}\right)$ is upper semicontinuous. This yields together with Lemma 3.3 that $\alpha \mapsto \gamma\left(Z^{\alpha}\right)$ is continuous ${ }^{2}$. The functional $\alpha \mapsto\left\langle R^{\alpha}, \mathcal{E}_{T}\right\rangle$ is linear and bounded and hence continuous. Consequently, the function

$$
\begin{aligned}
\alpha \mapsto \inf _{R \in \mathcal{D}}\left(E\left[Z^{\alpha} \int_{0}^{T} V\left(t, \frac{y Y_{t}^{R}}{Z_{t}^{\alpha}}\right) \mu(d t)\right]+y\left\langle R, \mathcal{E}_{T}\right\rangle\right)+\gamma\left(Z^{\alpha}\right) \\
=v_{Z^{\alpha}}(y)+\gamma\left(Z^{\alpha}\right)
\end{aligned}
$$

is also upper semicontinuous on $[0,1]$, therefore we get

$$
\begin{aligned}
& v(y)=\inf _{R \in \mathcal{D}}\left(E\left[Z^{1} \int_{0}^{T} V\left(t, \frac{y Y_{t}^{R^{1}}}{Z_{t}^{1}}\right) \mu(d t)\right]+y\left\langle R^{1}, \mathcal{E}_{T}\right\rangle\right)+\gamma\left(Z^{1}\right) \\
& \geq \underset{\alpha \uparrow 1}{\limsup }\left(v_{Z^{\alpha}}(y)+\gamma\left(Z^{\alpha}\right)\right) .
\end{aligned}
$$

This yields $v(y)=\inf _{Q \in \mathcal{Q}_{e}}\left(v_{Q}(y)+\gamma(Q)\right)$. Furthermore, observe that $v_{Q}(y)=\infty$ for $Q \in \mathcal{Q}_{e} \backslash \mathcal{Q}_{e}^{f}$. This follows again from [15, Lemma A.3] for $Q \sim P$ since then $v_{Q}$ and $u_{Q}$ satisfy the duality relations. We have $v(y) \leq \tilde{v}(y)$ as $\mathcal{D}_{Q} \subset \mathcal{D}$ for $Q \ll P$. Since $Z^{\alpha} \in \mathcal{Z}_{e}$ for $\alpha \in(0,1)$ we also get

$$
v(y) \geq \limsup _{\alpha \uparrow 1}\left(v_{Z^{\alpha}}(y)+\gamma\left(Z^{\alpha}\right)\right) \geq \inf _{Z \in \mathcal{Z}}\left(v_{Z}(y)+\gamma(Z)\right)=\tilde{v}(y) .
$$

This proves the first identity.

\footnotetext{
${ }^{2}$ For a more detailed argument compare [24, Remark 4.5].
} 


\section{Proofs for the primal problem}

In this section we will prove the missing assertions. First we make some easy observations. Due to (13) we know that for all $\alpha \in[0,1]$ we have

$$
\alpha \mathcal{A}\left(x_{1}, \mu\right)+(1-\alpha) \mathcal{A}\left(x_{2}, \mu\right) \subset \mathcal{A}\left(\alpha x_{1}+(1-\alpha) x_{2}, \mu\right) .
$$

Furthermore, if Assumption 2.4 is satisfied it is easy to show that, under the convention (5), $c \mapsto \mathcal{U}_{Q}(c)$ is a concave functional on $\mathcal{A}(x, \mu)$ for each $Q \in \mathcal{Q}$ and all $x>0$. These facts yield the concavity of the value functions $u_{Q}$ and $u$ and therefore under Assumption 2.4 the finiteness of the value function $u(x)$ for all $x>0$. The concavity of $u_{Q}$ implies in turn that

$$
u_{Q} \equiv+\infty \text { as soon as } E_{Q}\left[\int_{0}^{T} U^{+}\left(t, c_{t}\right) \mu(d t)\right]=+\infty \text { for some } c \in \bigcup_{x>0} \mathcal{A}(x, \mu) \text {. }
$$

Indeed, if $E_{Q}\left[\int_{0}^{T} U^{+}\left(t, c_{t}\right) \mu(d t)\right]=+\infty$ for $c \in \mathcal{A}(x, \mu)$ then it follows that $c+1 \in \mathcal{A}(x+1, \mu)$ and $E_{Q}\left[\int_{0}^{T} U\left(t, c_{t}\right) \mu(d t)\right]=+\infty$. Thus, $u_{Q}(x+1)=+\infty$. As $u_{Q}$ is concave this implies $u_{Q} \equiv+\infty$.

\section{Lemma 4.1}

We have the following minimax identity.

$$
\begin{aligned}
u(x) & =\sup _{c \in \mathcal{A}(x, \mu)} \inf _{Q \in \mathcal{Q}}\left(\mathcal{U}_{Q}(c)+\gamma(Q)\right)=\inf _{Q \in \mathcal{Q}}\left(u_{Q}(x)+\gamma(Q)\right) \\
& =\sup _{c \in \mathcal{A}(x, \mu)} \inf _{Q \in \mathcal{Q}_{e}}\left(\mathcal{U}_{Q}(c)+\gamma(Q)\right)=\inf _{Q \in \mathcal{Q}_{e}}\left(u_{Q}(x)+\gamma(Q)\right) .
\end{aligned}
$$

Hence, assertion 2 of Theorem 2.5 is valid.

Proof. Let $\varepsilon \in(0,1)$. Proposition 3.5 in [15] gives the existence of a utility function $\underline{U}$ such that $\underline{U}(x) \leq U(t, x)$ for all $x>0$ and all $t \in[0, T]$. With the help of this utility function we define $d=1+u(x+1)-\underline{U}(\varepsilon) \wedge 0$. Then we have

$$
u(x+1) \geq u(x+\varepsilon) \geq \sup _{c \in \mathcal{A}(x, \mu)} \inf _{Q \in \mathcal{Q}}\left(\mathcal{U}_{Q}(c+\varepsilon)+\gamma(Q)\right)=\sup _{c \in \mathcal{A}(x, \mu)} \inf _{Z \in \mathcal{Z}(d)}\left(\mathcal{U}_{Q}(c+\varepsilon)+\gamma(Z)\right) .
$$

Now $U(t, .+\varepsilon)$ is bounded from below and thus

$$
Z \mapsto E\left[Z \int_{0}^{T} U\left(t, c_{t}+\varepsilon\right) \mu(d t)\right]=\sup _{n} E\left[Z\left(\int_{0}^{T} U\left(t, c_{t}+\varepsilon\right) \mu(d t) \wedge n\right)\right]
$$

is a weakly lower semicontinuous affine functional. Lemma 3.3 states that $Z \mapsto \gamma(Z)$ is weakly lower semi-continuous and $\mathcal{Z}(d)$ is a weakly compact and convex set. Furthermore, for each $Z \in \mathcal{Z}(d)$ 
$c \mapsto \mathcal{U}_{Z}(c)$ is a concave functional defined on the convex set $\mathcal{A}(x, \mu)$. Therefore we may use the lopsided minimax theorem [1] to obtain

$$
\sup _{c \in \mathcal{A}(x, \mu)} \min _{Z \in \mathcal{Z}(d)}\left(\mathcal{U}_{Z}(c+\varepsilon)+\gamma(Z)\right)=\min _{Z \in \mathcal{Z}(d)} \sup _{c \in \mathcal{A}(x, \mu)}\left(\mathcal{U}_{Z}(c+\varepsilon)+\gamma(Z)\right)
$$

We know that these expressions are bounded by $u(x+\varepsilon)<d+\underline{U}(\varepsilon) \wedge 0$. Thus, it does not matter whether we take the infimum over $\mathcal{Z}$ or over $\mathcal{Z}(d)$. We obtain

$$
\begin{aligned}
u(x+\varepsilon) & \geq \inf _{Z \in \mathcal{Z}} \sup _{c \in \mathcal{A}(x, \mu)}\left(\mathcal{U}_{Z}(c+\varepsilon)+\gamma(Z)\right) \\
& \geq \inf _{Z \in \mathcal{Z}} \sup _{c \in \mathcal{A}(x, \mu)}\left(\mathcal{U}_{Z}(c)+\gamma(Z)\right) \\
& \geq \sup _{c \in \mathcal{A}(x, \mu)} \inf _{Z \in \mathcal{Z}}\left(\mathcal{U}_{Z}(c)+\gamma(Z)\right)=u(x) .
\end{aligned}
$$

As $\varepsilon \rightarrow 0$ the assertion follows since $u$ is continuous.

Observe now that Theorem 3.10 in [15] states that $u_{Q}(x)=\inf _{y>0}\left(v_{Q}(y)+x y\right)$ for all $Q \in \mathcal{Q}_{e}^{f}$. Pooling our lemmas and using this result, we get

$$
\begin{aligned}
u(x) & =\inf _{Q \in \mathcal{Q}_{e}}\left(u_{Q}(x)+\gamma(Q)\right)=\inf _{Q \in \mathcal{Q}_{e}^{f}}\left(u_{Q}(x)+\gamma(Q)\right) \\
& =\inf _{Q \in \mathcal{Q}_{e}^{f}} \inf _{y>0}\left(v_{Q}(y)+\gamma(Q)+x y\right)=\inf _{y>0}(v(y)+x y)
\end{aligned}
$$

which is assertion 3 of the theorem. Finiteness of $v$ and general duality principles yield then

$$
\lim _{x \rightarrow \infty} u(x) / x \rightarrow 0
$$

\section{Lemma 4.2}

For any $x>0$ there exist $\widehat{c} \in \mathcal{A}(x, \mu)$ such that

$$
\inf _{Q \in \mathcal{Q}}\left(E_{Q}\left[\int_{0}^{T} U\left(t, \widehat{c}_{t}\right) \mu(d t)\right]+\gamma(Q)\right)=u(x)
$$

Proof. Let $\left(c^{n}\right)$ be a maximizing sequence. Using again a Komlós-type argument we get a sequence $\left(\tilde{c}^{n}\right)_{n \in \mathbb{N}}$ with $\tilde{c}^{n} \in \operatorname{conv}\left(c^{n}, c^{n+1}, \ldots\right)$ converging $P \otimes \mu$-a.s. to some $\widehat{c} \in \mathcal{A}(x, \mu)$ since $\mathcal{A}(x, \mu)$ is closed under convergence in probability. The following adaption of the argument in [17] shows that the positive parts of $U\left(t, \tilde{c}_{t}^{n}\right)$ are uniformly integrable with respect to $Q \otimes \mu$ for all $Q \in Q_{e}^{f}$.

Assume $\left(U^{+}\left(t, \tilde{c}_{t}^{n}\right)\right)_{n}$ is not uniformly integrable. Then there is a constant $\alpha$, a subsequence which is also denoted $\left(\tilde{c}_{n}\right)_{n}$ and a partition $\left(A_{n}\right)$ such that $E\left[\int_{0}^{T} U^{+}\left(t, \tilde{c}_{t}^{n}\right) I_{A_{n}} \mu(d t)\right] \geq \alpha$ for 
$n \geq 1$. For $0 \leq t \leq T$ let $x_{t}^{0}=\inf \{x>0 \mid U(t, x) \geq 0\}$ and define a process $s^{n}=\left(s_{t}^{n}\right)_{0 \leq t \leq T}$ by $s_{t}^{n}=x_{t}^{0}+\sum_{k=1}^{n} \tilde{c}_{t}^{k}$. Hence, for any $R \in \mathcal{D}$ we have

$$
E\left[\int_{0}^{T} Y_{t}^{R} s_{t}^{n} \mu(d t)\right] \leq \int_{0}^{T} x_{t}^{0} \mu(d t)+n\left(x+\left\langle R, \mathcal{E}_{T}\right\rangle\right)
$$

This implies $s^{n} \in \mathcal{A}\left(\int_{0}^{T} x_{t}^{0} \mu(d t)+n x, \mu\right)$. Furthermore

$$
E\left[\int_{0}^{T} U\left(t, s_{t}^{n}\right) \mu(d t)\right] \geq \sum_{k=1}^{n} E\left[\int_{0}^{T} U^{+}\left(t, \tilde{c}_{t}^{k}\right) I_{A_{k}} \mu(d t)\right] \geq \alpha n .
$$

Which yields

$$
\begin{aligned}
\limsup _{x \rightarrow \infty} \frac{u(x)}{x} & \geq \limsup _{n \rightarrow \infty} \frac{E\left[\int_{0}^{T} U\left(t, s_{t}\right) \mu(d t)\right]}{\int_{0}^{T} x_{t}^{0} \mu(d t)+n x} \\
& \geq \limsup _{n \rightarrow \infty} \frac{\alpha n}{\int_{0}^{T} x_{t}^{0} \mu(d t)+n x} \\
& =\alpha>0
\end{aligned}
$$

in contradiction to (20).

Using the uniform integrability we can deduce that

$$
c \mapsto E_{Q}\left[\int_{0}^{T} U\left(t, c_{t}\right) \mu(d t)\right]+\gamma(Q)
$$

is upper semicontinuous with respect to almost sure convergence. From the concavity of

$$
c \mapsto \inf _{Q \in \mathcal{Q}_{e}^{f}}\left(E_{Q}\left[\int_{0}^{T} U\left(t, c_{t}\right) \mu(d t)\right]+\gamma(Q)\right)
$$

it follows that $\left(\tilde{c}^{n}\right)$ is still a maximizing sequence. Then the upper semicontinuity yields that

$$
\inf _{Q \in \mathcal{Q}_{e}}\left(E_{Q}\left[\int_{0}^{T} U\left(t, \widehat{c}_{t}\right) \mu(d t)\right]+\gamma(Q)\right) \geq u(x) .
$$

Actually we also have

$$
\inf _{Q \in \mathcal{Q}}\left(E_{Q}\left[\int_{0}^{T} U\left(t, \widehat{c}_{t}\right) \mu(d t)\right]+\gamma(Q)\right) \geq u(x):
$$

First we have that $\left\{Q \in \mathcal{Q} \mid E_{Q}\left[\int_{0}^{T} U\left(t, \widehat{c}_{t}\right) \mu(d t)\right]=-\infty\right\}=\emptyset$ since otherwise this set would have a nonempty intersection with $\mathcal{Q}_{e}$. Hence, for $Q \in \mathcal{Q} \backslash \mathcal{Q}_{e}, Q_{0} \in \mathcal{Q}_{e}^{f}$ and $Q_{\alpha}:=\alpha Q+(1-\alpha) Q_{0} \in \mathcal{Q}_{e}$, the limit of $E_{Q_{\alpha}}\left[\int_{0}^{T} U\left(t, \widehat{c}_{t}\right) \mu(d t)\right]$ as $\alpha \uparrow 1$ is $E_{Q}\left[\int_{0}^{T} U\left(t, \widehat{c}_{t}\right) \mu(d t)\right]$. Due to the convexity and lower semicontinuity of $\gamma$, we also have $\gamma\left(Q_{\alpha}\right) \rightarrow \gamma(Q)$. 


\section{Lemma 4.3}

The dual value function $v$ is lower semicontinuous.

Proof. Define $v(0)=\int_{0}^{T} \lim _{\varepsilon \rightarrow 0} V(t, \varepsilon) \mu(d t)$ and let $\left(y_{n}\right)_{n \in \mathbb{N}}$ be a sequence of positive reals converging to $y \geq 0$. If $\liminf _{n \rightarrow \infty} v\left(y_{n}\right)=\infty$ we are done. Otherwise we may assume that $\sup _{n} v\left(y_{n}\right)<\infty$. We proved that we have $\widehat{R}^{n} \in \mathcal{D}$ and $\widehat{Z}^{n} \in \mathcal{Z}$ such that

$$
v\left(y_{n}\right)=\mathcal{V}_{\widehat{Z}^{n}, \mu}\left(\frac{y_{n} Y^{\widehat{R}^{n}}}{\widehat{Z}^{n}}\right)+y_{n}\left\langle\widehat{R}^{n}, \mathcal{E}_{T}\right\rangle+\gamma\left(\widehat{Z}^{n}\right)
$$

And with an estimate similar to $(15)$ and $\mathcal{E}_{T} \geq 0$ we know that $v\left(y_{n}\right) \geq \int_{0}^{T} V\left(t, y_{n}\right) \mu(d t)+$ $\gamma\left(\widehat{Z}^{n}\right)$. Since $\gamma$ is bounded from below we get that $V\left(., y_{n}\right)<\infty \widehat{Q}^{n} \otimes \mu$-a.s., where $d \widehat{Q}^{n}=$ $\widehat{Z}^{n} d P$. Furthermore we can find $d$ such that $\widehat{Z}_{n} \in \mathcal{Z}(d)$. This enables us to use once more a Komlós-type argument to get a sequence of convex combinations, $\left(\left(\tilde{R}^{n}, \tilde{Z}^{n}\right)\right)_{n}$ where $\left(\tilde{R}^{n}, \tilde{Z}^{n}\right) \in$ $\operatorname{conv}\left\{\left(R^{n}, Z^{n}\right),\left(R^{n+1}, Z^{n+1}\right), \ldots\right\}$, which converges $P$-a.s. to some $(\widehat{R}, \widehat{Z})$. Now we use Lemma 3.3 and the convexity of $(Z, R) \mapsto Z \int_{0}^{T} V\left(t, y Y_{t}^{R} / Z_{t}\right) \mu(d t)$ to obtain

$$
v(y) \leq \mathcal{V}_{\widehat{Z}, \mu}\left(\frac{y Y^{\widehat{R}}}{\widehat{Z}}\right)+y\left\langle\widehat{R}, \mathcal{E}_{T}\right\rangle+\gamma(\widehat{Z}) \leq \liminf _{n \rightarrow \infty} v\left(y_{n}\right)
$$

which is the desired lower semicontinuity.

Proof of Theorem 2.5. Assertion 2 and 3, the finiteness of $u$ and $v$, and the concavity of $u$ were already proved. The convexity of $v$ is an immediate consequence of the convexity of $\left(Z, y Y^{R}\right) \mapsto \mathcal{V}_{Z}\left(Y^{R} / Z\right)+\gamma(Z)$. Furthermore, the lower semicontinuity of $v$ (see Lemma 4.3) implies assertion 3 of Theorem 2.5. Equation (10) follows as in [15, Lemma A.7]. If $\mathcal{E}_{T} \equiv 0$ this implies $v^{\prime}(\infty-)=0$ which yields by general duality results that $u^{\prime}(0)=\infty$ which is equation (11). Assertion 5 corresponds to Lemma 3.5. Furthermore, the existence of an optimal $c$ (item 6) is the content of Lemma 4.2 .

We will now deal with the existence of a saddle point. Let $y>0$ be such that $v(y)+y x=u(x)$, such a $y$ exists due to the behavior of $v^{\prime}$. Take then a solution $(\widehat{R}, \widehat{Q})$ to the dual problem for $y$ and let $\widehat{Z}$ be the density of $\widehat{Q}$ with respect to $P$. Let $Z^{1}$ be in $\mathcal{Z}_{e}^{f}$ and define $Z^{\alpha}=\alpha Z^{1}+(1-\alpha) \widehat{Z}$ for $\alpha \in(0,1]$. Then $v_{Z^{\alpha}}(y)+\gamma\left(Z^{\alpha}\right) \rightarrow v(y)$ as $\alpha \rightarrow 0$ : Let $R^{1}$ be such that

$$
\mathcal{V}_{Z^{1}}\left(y Y^{R^{1}} / \widehat{Z}^{1}\right)+\left\langle R^{1}, \mathcal{E}_{T}\right\rangle=v_{Z^{1}}(y)
$$


Then define $R^{\alpha}=\alpha R^{1}+(1-\alpha) \widehat{R}$. We get

$$
\begin{aligned}
v(y) & \leq v_{Z^{\alpha}}(y)+\gamma\left(Z^{\alpha}\right) \\
& \leq \mathcal{V}_{Z^{\alpha}}\left(y Y^{R^{\alpha}} / Z^{\alpha}\right)+\left\langle R^{\alpha}, \mathcal{E}_{T}\right\rangle+\gamma\left(Z^{\alpha}\right) \\
& \leq \alpha\left(v_{Z^{1}}(y)+\gamma\left(Z^{1}\right)\right)+(1-\alpha)\left(\tilde{v}_{\widehat{Z}}(y)+\gamma(\widehat{Z})\right)
\end{aligned}
$$

because of convexity. Observe that the righthand side of the equation goes to $v(y)$ as $\alpha$ goes to 0 .

Due to the duality relations we have that $v_{Z^{\alpha}}+x y \geq u_{Z^{\alpha}}$ and $u_{Z^{\alpha}}+\gamma\left(Z^{\alpha}\right) \rightarrow u_{\widehat{Z}}+\gamma(\widehat{Z})$. Hence,

$$
\begin{aligned}
u(x) & =v(y)+y x=\lim _{\alpha \rightarrow 0}\left(v_{Z^{\alpha}}(y)+x y+\gamma\left(Z^{\alpha}\right)\right) \\
& \geq \lim _{\alpha \rightarrow 0}\left(u_{Z^{\alpha}}(x)+\gamma\left(Z^{\alpha}\right)\right)=u_{\widehat{Z}}(x)+\gamma(\widehat{Z})
\end{aligned}
$$

With the minimax identity we get $u(x)=u_{\widehat{Z}}+\gamma(\widehat{Z})$ and therefore we get for $\hat{c}$ as in Lemma 4.2

$$
u(x)=u_{\widehat{Z}}(x)+\gamma(\widehat{Z}) \geq \mathcal{U}_{\widehat{Z}, \mu}(\hat{c})+\gamma(\widehat{Z}) \geq \inf _{Q \in \mathcal{Q}} \mathcal{U}_{Q}(\hat{c})+\gamma(\widehat{Z})=u(x) .
$$

Now we show that $\hat{c}=I\left(\cdot, y Y^{\widehat{R}}\right) \widehat{Q} \otimes \mu$-a.s. We have

$$
0 \leq V\left(t, \frac{\widehat{y} Y_{t}^{\widehat{R}}}{\widehat{Z}_{t}}\right)+\frac{\widehat{y} Y_{t}^{\widehat{R}}}{\widehat{Z}_{t}} \hat{c}_{t}-U\left(t, \hat{c}_{t}\right)
$$

and therefore

$$
\begin{aligned}
0 & \leq E_{\widehat{Q}}\left[\int_{0}^{T} V\left(t, \frac{y Y_{t}^{\widehat{R}}}{\widehat{Z}_{t}}\right) \mu(d t)+\int_{0}^{T} \frac{y Y_{t}^{\widehat{R}}}{\widehat{Z}_{t}} \hat{c}_{t} \mu(d t)-\int_{0}^{T} U\left(t, \hat{c}_{t}\right) \mu(d t)\right] \\
& =v(y)+E\left[\int_{0}^{T} y Y_{t}^{\widehat{R}} \hat{c}_{t} \mu(d t)\right]-y\left\langle\widehat{R}, \mathcal{E}_{T}\right\rangle-u(x) \\
& \leq v(y)+y x-u(x)=0 .
\end{aligned}
$$

Together this implies

$$
0=V\left(t, \frac{y Y_{t}^{\widehat{R}}}{\widehat{Z}_{t}}\right)+\frac{y Y_{t}^{\widehat{R}}}{\widehat{Z}_{t}} \hat{c}_{t}-U\left(t, \hat{c}_{t}\right) \quad \widehat{Q} \otimes \mu(d t) \text {-a.s. }
$$

which means that $\hat{c}_{t}=I\left(t, y Y_{t}^{\widehat{R}} / \widehat{Z}_{t}\right)$.

The fact that $u^{\prime}(\infty-)=0$ follows from (20). Therefore we also have $v^{\prime}(0+)=-\infty$. To obtain the strict concavity of the function $u$ (and hence the differentiability of $v$ ) assume that $u$ is not strictly concave. Since $u$ is increasing and because of the conditions for the derivatives we know there exist $0<x_{0}<x_{1}$ and $y>0$ such that $v(y)+y x_{0}=u\left(x_{0}\right)$ and $v(y)+y x_{1}=u\left(x_{1}\right)$. Let $c^{0}, c^{1}$ be the corresponding optimal consumption processes and $(\widehat{Q}, \widehat{R})$ the solution to the dual problem. Then we have $c_{t}^{0}=I\left(t, y Y^{\widehat{R}}\right)=c_{t}^{1} \widehat{Q}$-a.s. and $E_{\widehat{Q}}\left[\int_{0}^{T} c_{t}^{0} y Y_{t}^{\widehat{R}} \mu(d t)\right]+y\left\langle\widehat{R}, \mathcal{E}_{T}\right\rangle=x_{0} y<x_{1} y=$ $E_{\widehat{Q}}\left[\int_{0}^{T} c_{t}^{1} y Y_{t}^{\widehat{R}} \mu(d t)\right]+y\left\langle\widehat{R}, \mathcal{E}_{T}\right\rangle$. This is a contradiction. 
Proof of Corollary 2.6. This corollary can be proved by copying the arguments of the proof of Proposition 2.5 in [24].

\section{References}

[1] J.-P. Aubin and I. Ekeland. Applied nonlinear analysis, chapter 6. John Wiley \& Sons, New York, 1984.

[2] D. Becherer. Bounded solutions to backward SDE's with jumps for utility optimization and indifference hedging. Annals of Applied Probability, 2006.

[3] G. Bordigoni, A. Matoussi, and M. Schweizer. A stochastic control approach to a robust utility maximization problem. In Proceedings of Abel Symposium 2005. Springer.

[4] C. Burgert and L. Rüschendorf. Optimal consumption strategies under model uncertainty. Statistics \& Decisions, 2004.

[5] J. Cvitanić, W. Schachermayer, and H. Wang. Utility maximization in incomplete markets with random endowment. Finance \& Stochastics, 5(2):259-272, 2001.

[6] F. Delbaen and W. Schachermayer. A general version of the fundamental theorem of asset pricing. Mathematische Annalen, 300(3):463-520, 1994.

[7] D. Duffie and T. Zariphopoulou. Optimal investment with undiversifiable income risk. Mathematical Finance, 3:135-148, 1993.

[8] H. Föllmer and D. Kramkov. Optional decompositions under constraints. Probability Theory Related Fields, 109(1):1-25, 1997.

[9] I. Gilboa and D. Schmeidler. Maxmin expected utility with non-unique prior. Journal of Mathematical Economics, (18):141-153, 1989.

[10] A. Gundel. Robust utility maximization for complete and incomplete market models. Finance and Stochastics, 9(2):151-176, 2005.

[11] L. P. Hansen and T. J. Sargent. Robust control and model uncertainty. American Economic Review, 91:60-66, 2001. 
[12] D. Hernández-Hernández and A. Schied. A control approach to robust utility maximization with logarithmic utility and time consistent penalties. submitted, 2006.

[13] D. Hernández-Hernández and A. Schied. Robust utility maximization in a stochastic factor model. to appear in Statistics \& Decisions 24, Special Volume on Risk Measures, 2006.

[14] J. Hugonnier and D. Kramkov. Optimal investment with random endowments in incomplete markets. Annals of Applied Probability, 14(2):845-864, 2004.

[15] I. Karatzas and G. Žitković. Optimal consumption from investment and random endowment in incomplete semimartingale markets. Annals of Probability, 31(4):1821-1858, 2003.

[16] D. Kramkov and W. Schachermayer. The asymptotic elasticity of utility functions and optimal investment in incomplete markets. Annals of Applied Probability, 9(3):904-950, 1999.

[17] D. Kramkov and W. Schachermayer. Necessary and sufficient conditions in the problem of optimal investment in incomplete markets. Annals of Applied Probability, 13(4):1504-1516, 2003.

[18] F. Maccheroni, M. Marinacci, and A. Rustichini. Ambiguity aversion, malevolent nature, and the variational representation of preferences. Preprint, 2004.

[19] R. C. Merton. Lifetime portfolio selection under uncertainty: The continuous-time case. Review of Economics and Statistics, 1969.

[20] R. C. Merton. Optimum consumption and portfolio rules in a continuous time model. Journal of Economic Theory, 3:373-413, 1971.

[21] M. Müller. Market completion and robust utility maximization. PhD thesis, Humboldt University, 2005.

[22] M.-C. Quenez. Optimal portfolio in a multiple-priors model. In Seminar on stochastic analysis, random fields and applications IV, Progress in Probability 58, pages 291-321, Basel, 2004. Birkhäuser.

[23] A. Schied. On the Neyman-Pearson problem for law-invariant risk measures and robust utility functionals. Annals of Applied Probability, 14:1398-1423, 2004. 
[24] A. Schied. Optimal investments for risk- and ambiguity-averse preferences: a duality approach. Preprint, 2005.

[25] A. Schied and C.-T. Wu. Duality theory for optimal investments under model uncertainty. Statistics \&3 Decisions, 23(3):199-217, 2005. 


\section{SFB 649 Discussion Paper Series 2006}

For a complete list of Discussion Papers published by the SFB 649, please visit http://sfb649.wiwi.hu-berlin.de.

001 "Calibration Risk for Exotic Options" by Kai Detlefsen and Wolfgang K. Härdle, January 2006.

002 "Calibration Design of Implied Volatility Surfaces" by Kai Detlefsen and Wolfgang K. Härdle, January 2006.

003 "On the Appropriateness of Inappropriate VaR Models" by Wolfgang Härdle, Zdeněk Hlávka and Gerhard Stahl, January 2006.

004 "Regional Labor Markets, Network Externalities and Migration: The Case of German Reunification" by Harald Uhlig, January/February 2006.

005 "British Interest Rate Convergence between the US and Europe: A Recursive Cointegration Analysis" by Enzo Weber, January 2006.

006 "A Combined Approach for Segment-Specific Analysis of Market Basket Data" by Yasemin Boztuğ and Thomas Reutterer, January 2006.

007 "Robust utility maximization in a stochastic factor model" by Daniel Hernández-Hernández and Alexander Schied, January 2006.

008 "Economic Growth of Agglomerations and Geographic Concentration of Industries - Evidence for Germany" by Kurt Geppert, Martin Gornig and Axel Werwatz, January 2006.

009 "Institutions, Bargaining Power and Labor Shares" by Benjamin Bental and Dominique Demougin, January 2006.

010 "Common Functional Principal Components" by Michal Benko, Wolfgang Härdle and Alois Kneip, Jauary 2006.

011 "VAR Modeling for Dynamic Semiparametric Factors of Volatility Strings" by Ralf Brüggemann, Wolfgang Härdle, Julius Mungo and Carsten Trenkler, February 2006.

012 "Bootstrapping Systems Cointegration Tests with a Prior Adjustment for Deterministic Terms" by Carsten Trenkler, February 2006.

013 "Penalties and Optimality in Financial Contracts: Taking Stock" by Michel A. Robe, Eva-Maria Steiger and Pierre-Armand Michel, February 2006.

014 "Core Labour Standards and FDI: Friends or Foes? The Case of Child Labour" by Sebastian Braun, February 2006.

015 "Graphical Data Representation in Bankruptcy Analysis" by Wolfgang Härdle, Rouslan Moro and Dorothea Schäfer, February 2006.

016 "Fiscal Policy Effects in the European Union" by Andreas Thams, February 2006.

017 "Estimation with the Nested Logit Model: Specifications and Software Particularities" by Nadja Silberhorn, Yasemin Boztuğ and Lutz Hildebrandt, March 2006.

018 "The Bologna Process: How student mobility affects multi-cultural skills and educational quality" by Lydia Mechtenberg and Roland Strausz, March 2006.

019 "Cheap Talk in the Classroom" by Lydia Mechtenberg, March 2006.

020 "Time Dependent Relative Risk Aversion" by Enzo Giacomini, Michael Handel and Wolfgang Härdle, March 2006.

021 "Finite Sample Properties of Impulse Response Intervals in SVECMs with Long-Run Identifying Restrictions" by Ralf Brüggemann, March 2006.

022 "Barrier Option Hedging under Constraints: A Viscosity Approach" by Imen Bentahar and Bruno Bouchard, March 2006.

\section{SFB 649, Spandauer Straße 1, D-10178 Berlin} http:/ / sfb649.wiwi.hu-berlin.de

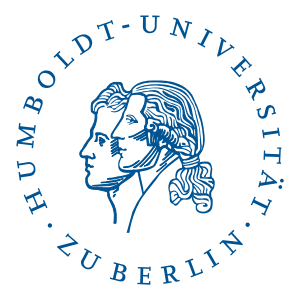


023 "How Far Are We From The Slippery Slope? The Laffer Curve Revisited" by Mathias Trabandt and Harald Uhlig, April 2006.

024 "e-Learning Statistics - A Selective Review" by Wolfgang Härdle, Sigbert Klinke and Uwe Ziegenhagen, April 2006.

025 "Macroeconomic Regime Switches and Speculative Attacks" by Bartosz Maćkowiak, April 2006.

026 "External Shocks, U.S. Monetary Policy and Macroeconomic Fluctuations in Emerging Markets" by Bartosz Maćkowiak, April 2006.

027 "Institutional Competition, Political Process and Holdup" by Bruno Deffains and Dominique Demougin, April 2006.

028 "Technological Choice under Organizational Diseconomies of Scale" by Dominique Demougin and Anja Schöttner, April 2006.

029 "Tail Conditional Expectation for vector-valued Risks" by Imen Bentahar, April 2006.

030 "Approximate Solutions to Dynamic Models - Linear Methods" by Harald Uhlig, April 2006.

031 "Exploratory Graphics of a Financial Dataset" by Antony Unwin, Martin Theus and Wolfgang Härdle, April 2006.

032 "When did the 2001 recession really start?" by Jörg Polzehl, Vladimir Spokoiny and Cătălin Stărică, April 2006.

033 "Varying coefficient GARCH versus local constant volatility modeling. Comparison of the predictive power" by Jörg Polzehl and Vladimir Spokoiny, April 2006.

034 "Spectral calibration of exponential Lévy Models [1]" by Denis Belomestny and Markus Reiß, April 2006.

035 "Spectral calibration of exponential Lévy Models [2]" by Denis Belomestny and Markus Reiß, April 2006.

036 "Spatial aggregation of local likelihood estimates with applications to classification" by Denis Belomestny and Vladimir Spokoiny, April 2006.

037 "A jump-diffusion Libor model and its robust calibration" by Denis Belomestny and John Schoenmakers, April 2006.

038 "Adaptive Simulation Algorithms for Pricing American and Bermudan Options by Local Analysis of Financial Market" by Denis Belomestny and Grigori N. Milstein, April 2006.

039 "Macroeconomic Integration in Asia Pacific: Common Stochastic Trends and Business Cycle Coherence" by Enzo Weber, May 2006.

040 "In Search of Non-Gaussian Components of a High-Dimensional Distribution" by Gilles Blanchard, Motoaki Kawanabe, Masashi Sugiyama, Vladimir Spokoiny and Klaus-Robert Müller, May 2006.

041 "Forward and reverse representations for Markov chains" by Grigori N. Milstein, John G. M. Schoenmakers and Vladimir Spokoiny, May 2006.

042 "Discussion of 'The Source of Historical Economic Fluctuations: An Analysis using Long-Run Restrictions' by Neville Francis and Valerie A. Ramey" by Harald Uhlig, May 2006.

043 "An Iteration Procedure for Solving Integral Equations Related to Optimal Stopping Problems" by Denis Belomestny and Pavel V. Gapeev, May 2006.

044 "East Germany's Wage Gap: A non-parametric decomposition based on establishment characteristics" by Bernd Görzig, Martin Gornig and Axel Werwatz, May 2006.

045 "Firm Specific Wage Spread in Germany - Decomposition of regional differences in inter firm wage dispersion" by Bernd Görzig, Martin Gornig and Axel Werwatz, May 2006.

\section{SFB 649, Spandauer Straße 1, D-10178 Berlin http:/ / sfb649.wiwi.hu-berlin.de}

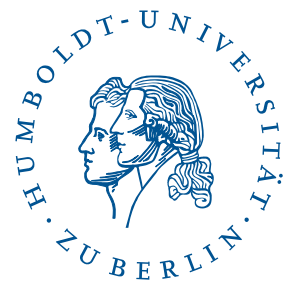


046 "Produktdiversifizierung: Haben die ostdeutschen Unternehmen den Anschluss an den Westen geschafft? - Eine vergleichende Analyse mit Mikrodaten der amtlichen Statistik" by Bernd Görzig, Martin Gornig and Axel Werwatz, May 2006.

047 "The Division of Ownership in New Ventures" by Dominique Demougin and Oliver Fabel, June 2006.

048 "The Anglo-German Industrial Productivity Paradox, 1895-1938: A Restatement and a Possible Resolution" by Albrecht Ritschl, May 2006.

049 "The Influence of Information Costs on the Integration of Financial Markets: Northern Europe, 1350-1560" by Oliver Volckart, May 2006.

050 "Robust Econometrics" by Pavel Čížek and Wolfgang Härdle, June 2006.

051 "Regression methods in pricing American and Bermudan options using consumption processes" by Denis Belomestny, Grigori N. Milstein and Vladimir Spokoiny, July 2006.

052 "Forecasting the Term Structure of Variance Swaps" by Kai Detlefsen and Wolfgang Härdle, July 2006.

053 "Governance: Who Controls Matters" by Bruno Deffains and Dominique Demougin, July 2006.

054 "On the Coexistence of Banks and Markets" by Hans Gersbach and Harald Uhlig, August 2006.

055 "Reassessing Intergenerational Mobility in Germany and the United States: The Impact of Differences in Lifecycle Earnings Patterns" by Thorsten Vogel, September 2006.

056 "The Euro and the Transatlantic Capital Market Leadership: A Recursive Cointegration Analysis" by Enzo Weber, September 2006.

057 "Discounted Optimal Stopping for Maxima in Diffusion Models with Finite Horizon" by Pavel V. Gapeev, September 2006.

058 "Perpetual Barrier Options in Jump-Diffusion Models" by Pavel V. Gapeev, September 2006.

059 "Discounted Optimal Stopping for Maxima of some Jump-Diffusion Processes" by Pavel V. Gapeev, September 2006.

060 "On Maximal Inequalities for some Jump Processes" by Pavel V. Gapeev, September 2006.

061 "A Control Approach to Robust Utility Maximization with Logarithmic Utility and Time-Consistent Penalties" by Daniel Hernández-Hernández and Alexander Schied, September 2006.

062 "On the Difficulty to Design Arabic E-learning System in Statistics" by Taleb Ahmad, Wolfgang Härdle and Julius Mungo, September 2006.

063 "Robust Optimization of Consumption with Random Endowment" by Wiebke Wittmüß, September 2006.

SFB 649, Spandauer Straße 1, D-10178 Berlin http:/ / sfb649.wiwi.hu-berlin.de 